\title{
Initial on-orbit radiometric calibration of the Suomi NPP VIIRS reflective solar bands
}

\author{
Ning Lei ${ }^{1, *}$, Zhipeng Wang ${ }^{1}$, Jon Fulbright ${ }^{1}$, Shihyan Lee ${ }^{1}$, Jeff McIntire ${ }^{1}$, Vincent \\ Chiang ${ }^{1}$, and Jack Xiong ${ }^{2}$
}

1. Sigma Space Corporation, 4600 Forbes Blvd., Lanham, MD, USA 20706

2. Sciences and Exploration Directorate, NASA Goddard Space Flight Center, Greenbelt, MD, USA 20771

\begin{abstract}
The on-orbit radiometric response calibration of the VISible/Near InfraRed (VISNIR) and the Short-Wave InfraRed (SWIR) bands of the Visible/Infrared Imager/Radiometer Suite (VIIRS) aboard the Suomi National Polar-orbiting Partnership (NPP) satellite is carried out through a Solar Diffuser (SD). The transmittance of the SD screen and the SD’s Bidirectional Reflectance Distribution Function (BRDF) are measured before launch and tabulated, allowing the VIIRS sensor aperture spectral radiance to be accurately determined. The radiometric response of a detector is described by a quadratic polynomial of the detector's digital number (dn). The coefficients were determined before launch. Once on orbit, the coefficients are assumed to change by a common factor: the F-factor. The radiance scattered from the SD allows the determination of the F-factor. In this Proceeding, we describe the methodology and the associated algorithms in the determination of the F-factors and discuss the results.
\end{abstract}

Keywords: remote sensing, radiance, calibration, VIIRS, RSB, radiometric, Solar Diffuser, F-factor, degradation, tungsten oxide

\section{INTRODUCTION}

The Visible Infrared Imaging Radiometer Suite (VIIRS) aboard the Suomi NPP satellite (launched on October $28,2011)$ is the next generation polar-orbiting remote sensing whisk-broom scanner to the MODerate Resolution Imaging Spectroradiometer (MODIS), providing imaging capabilities in 22 spectral bands over a wavelength range of $0.4 \mu \mathrm{m}$ to $12.0 \mu \mathrm{m}$. The design specified and the measured band central wavelengths and widths can be found in Ref. 1. Although carefully calibrated before launch, the on-orbit VIIRS sensor's radiometric response can change substantially with time as seen in MODIS ${ }^{2}$. Consequently, on-orbit radiometric calibration for the VIIRS sensor is critical for generating high quality weather and environmental products. In this Proceeding, we give the methodology and the results of the on-orbit calibration of the Reflective Solar Bands (RSB). Our results are used to validate the results from the Aerospace Corp.

\section{METHOD FOR THE ON-ORBIT RADIOMETRIC RESPONSE CALIBRATION}

The primary on-orbit radiometric calibration in the RSB for NPP VIIRS is through the onboard Solar Diffuser (SD). Fig. 1 is a schematic showing the onboard RSB calibration physical components. When fully illuminated by the Sun through a pinhole attenuation screen, the SD provides a known spectral radiance source over a wide spectrum covering the visible and near infrared wavelengths by scattering the incident solar radiation. As a result, 
viewing the fully illuminated SD allows the determination of a detector's response coefficient at the particular spectral radiance level provided by the SD. The validity of the coefficient is then extended to other radiance levels.

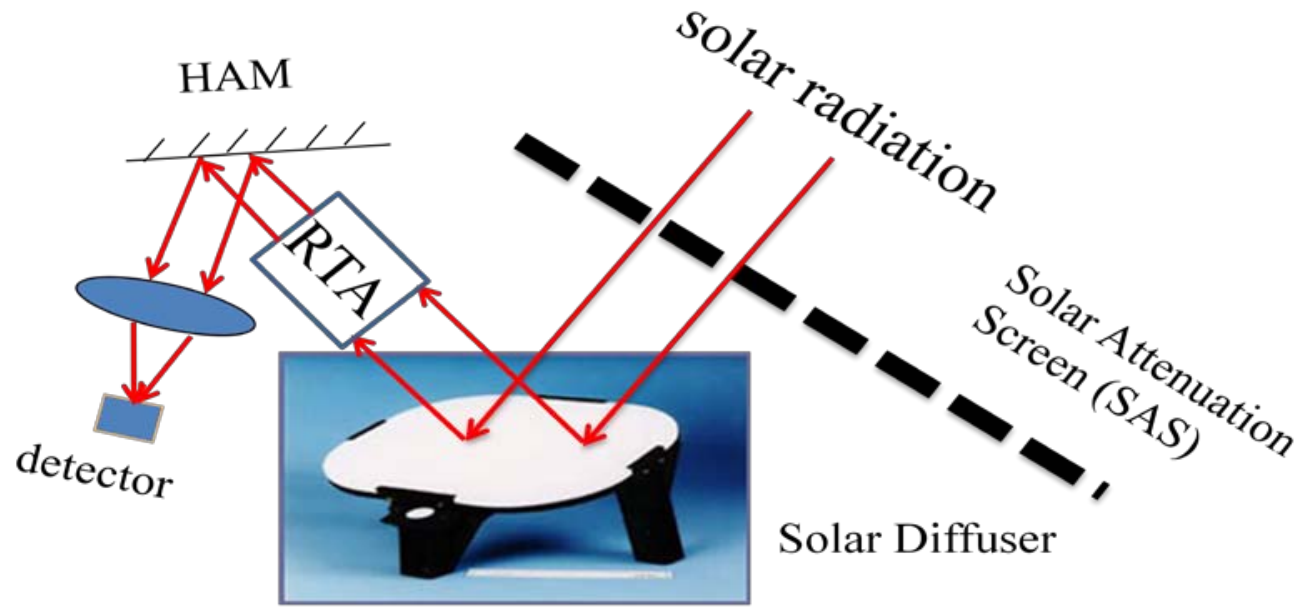

Fig. 1. A schematic shows the observation of the SD scattered solar radiation.

We denote the response coefficient as $F$, used as a multiplication factor in the following equation

$L_{S D}=F \times\left(c_{0}+c_{1} * d n+c_{2} * d n^{2}\right)$,

where $d n$ is a detector's digital number with background subtracted. In Eq. (1), $\left(c_{0}, c_{1}, c_{2}\right)$ are determined through linear interpolations in the temperature of the electronics and in the temperature of the VISNIR focal plane for the VISNIR bands and the Optical Mechanics Module for the SWIR bands using prelaunch determined $\left(c_{0}, c_{1}, c_{2}\right)^{3}$ at three temperature plateaus. $L_{S D}$ is the solar spectral radiance scattered from the $\mathrm{SD}$, computed by

$L_{S D}=\frac{\Phi_{\text {solar spectral power }}}{4 \pi * d_{\text {VIIRS-sun }}^{2}} \times \tau_{S A S} \times \cos \theta_{S D-\text { sun }} \times B R D F \times R V S$

Eq. (2) is critical to the computation of the F-factor defined in Eq. (1) and needs careful explanations. In Eq. (2), $\Phi_{\text {solar spectral power }}$ is the solar spectral power at a distance of one astronomical unit, a wavelength-dependent

predetermined time-independent quantity (the time independence is an approximation). $\tau_{S A S}$ is the transmittance of the pinhole screen for the SD, to allow the SD to provide spectral radiances close to the typical radiances for the RSB bands. Due to the pinholes, $\tau_{S A S}$ is dependent on the incident solar angle and can also be weakly dependent on the wavelength due to pinhole inner surface total reflection. As shown in Fig. 2, the incident solar angle components are quantified by a projected vertical angle $\phi_{V}$ in the XZ plane and a horizontal angle $\phi_{H}$ in the XY plane in VIIRS coordinate system. The $\mathrm{X}$ axis is along the longitudinal direction of VIIRS and the $\mathrm{Z}$ axis points to the EV port. Mathematically, the angles are computed by

$\phi_{H}=-\arctan (y / x)$,

and 


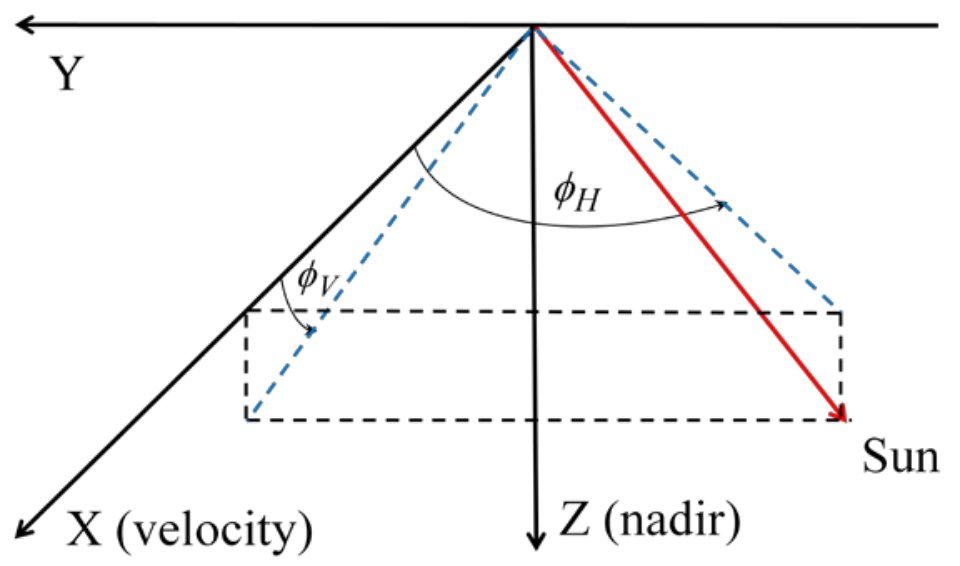

Fig. 2. Definitions of the projected vertical and horizontal angles.

$\tau_{S A S}$ is determined before launch. $\theta_{S D \text {-sun }}$ is the angle between the normal of the SD surface and the SD-Sun vector. $B R D F$ is the Bidirectional Reflectance Distribution Function of the SD, a function of both the incident solar angle and the VIIRS sensor's SD view angle as well as wavelength and time. Since the VIIRS sensor's SD view angle is time invariant, $B R D F$ may be expressed as

$B R D F=H\left(\lambda, t-t_{0}\right) \times B R D F\left(\lambda, t=t_{0} ; \phi_{H}, \phi_{V}\right)$,

where $H\left(\lambda, t-t_{0}\right)$ represents the deterioration of the SD due to solar radiation, $t_{0}$ is the starting time when the SD is initially exposed to the Sun, and $\operatorname{BRDF}\left(\lambda, t=t_{0} ; \phi_{H}, \phi_{V}\right)$ is the initial $B R D F$ of the SD, determined before launch. $H\left(\lambda, t-t_{0}\right)$ is determined by an onboard optical device which views the Sun (through a pinhole screen) and the SD at almost the same time. RVS (Response Versus Scan) is the reflectivity of the Half-Angle-Mirror (HAM) of VIIRS, an incident angle dependent quantity ${ }^{4}$. The HAM is an integral part of the sensor, residing right after the Rotating Telescope Assembly (RTA) in the optical path. RVS is weakly wavelength dependent for the RSB. Note that unlike other components in the sensor's optical path, the HAM's reflectivity is treated independently because as the HAM scans the EV port, the incident angle varies.

Before we can use Eq. (1) to compute the F-factor, we need to pay attention to a detector's ability to convert incident photons to electrons over a wide range of wavelengths. This ability is quantified by the Relative Spectral Response function ${ }^{1}$, denoted by $\operatorname{RSR}(\lambda, B)$ with $B$ denoting a particular band. With $R S R$, Eq. (1) is modified to be

$$
F=\frac{\int R S R(\lambda, B) \times L_{S D}(\lambda) d \lambda}{\left(c_{0}+c_{1} * d n+c_{2} * d n^{2}\right) \times \int R S R(\lambda, B) d \lambda} .
$$

\section{SWEET SPOT DETERMINATION}

When the SD is fully illuminated by the Sun, seen by the VIIRS detectors, the solar vector angle components $\phi_{H}$ and $\phi_{V}$ are said to be in the sweet spot. VIIRS is such designed that when at the sweet spot, the $\mathrm{Z}$ axis points at part of the Earth which is dark, avoiding complicated earth shine through the EV port in the F-factor determination. In addition, the louvers of the SD screen are designed to reduce earth shine on the SD. The sweet spot is determined 
by plotting the DN (not background subtracted $\mathrm{dn}$ ) of the detectors versus the projected vertical solar angle $\phi_{V}$. A set of plots of DN versus $\phi_{V}$ is shown in Figs. 3a-b for orbits 154 (2011-11-08) and 3272 (2012-06-15).
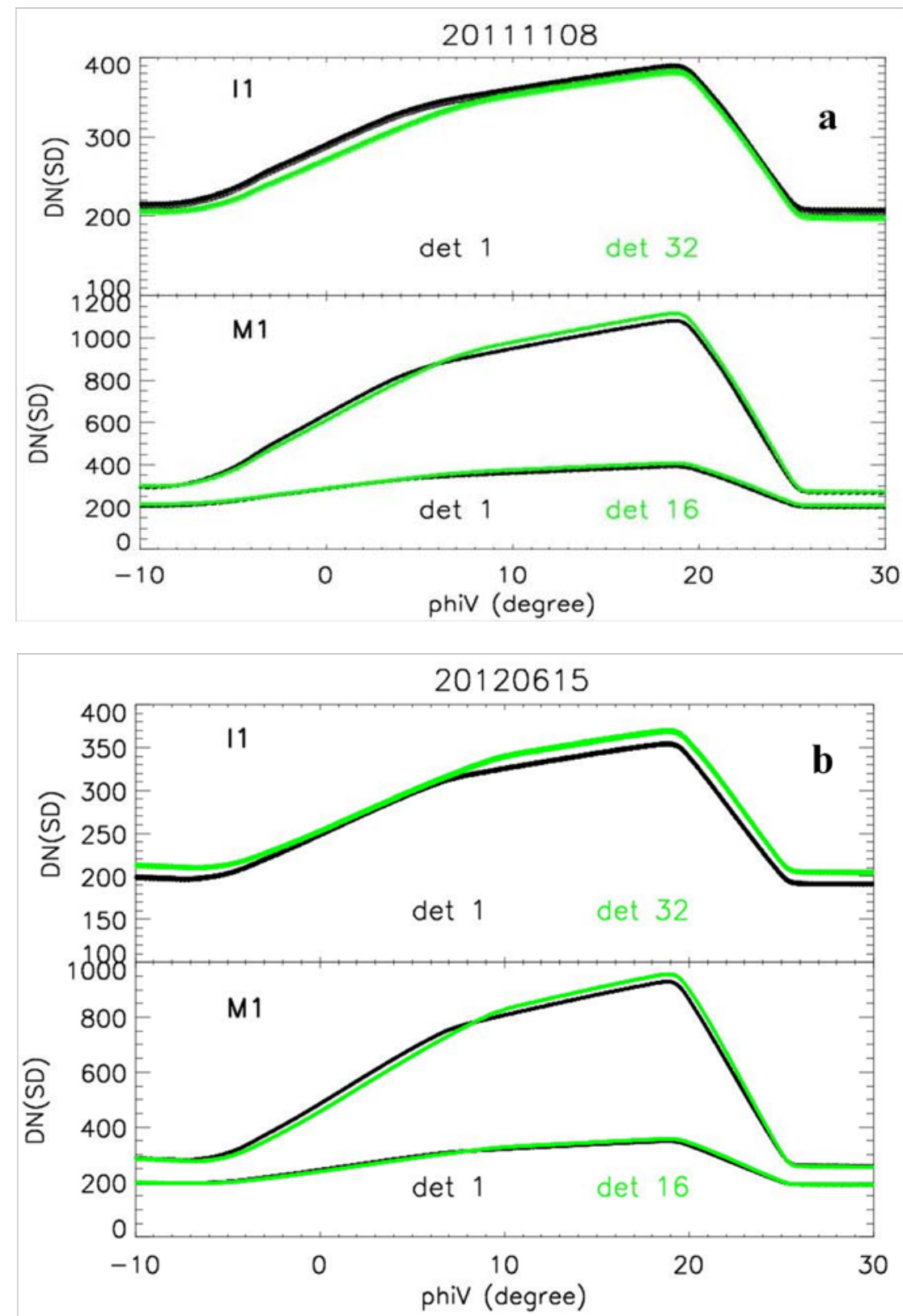

Fig. 3. Plots of DN(SD) (not background subtracted) versus $\phi_{V}$ for orbit 154 (a) and orbit 3272 (b) for the first and the last detectors of bands I1 and M1 respectively, with $\phi_{H} \approx 28.0^{\circ}$ (orbit 154) and $\phi_{H} \approx 15.5^{\circ}$ (orbit 3272), respectively. The lower curves for band M1 are for the low gain state and the higher curves for the high gain state.

We can see in Figs. 3a-b that when $\phi_{V}$ is greater than 19 degrees, DN drops very quickly with increasing angle, a result of earth shadow covering the SD; and when $\phi_{V}$ is less than about 6 degrees, DN drops quickly with decreasing angle, due to the moving out-of-detector-sight of the solar illumination on the SD at smaller $\phi_{V}$. From 
Fig. 3, the sweet spot is chosen to be $\phi_{V}$ in the region of [14.0, 18.0] degrees (the lower limit of the sweet sport can be relaxed to be a smaller angle). The sweet spot is much less sensitive to the horizontal angle $\phi_{H}$ evidenced by the similarity of the plots although the corresponding horizontal angles are quite different (at orbit $154 \phi_{H} \approx 28.0^{\circ}$ and at orbit $3272 \phi_{H} \approx 15.5^{\circ}$ ). The lower curves for band M1 in Figs. 3a-b are for the low gain state.

\section{RESULTS}

After the degassing phase, NPP VIIRS began operation mode on November 8, 2011 on orbit 154, allowing the start of the F-factor determination for the VISible and Near InfraRed (VISNIR) bands in the RSB. The F-factor determination for the Short Wave InfraRed (SWIR) bands in the RSB started after the cryo-door opened a few days prior to orbit 1201 to cool down the SWIR band focal plane.

For each orbit, there are a few dozen scans satisfying the sweet spot criterion, with each of the scans lasting nominally 1.7864 seconds. We compute the F-factor for each scan and then compute the simple average per orbit through a repeated 4-sigma outlier rejection procedure. We compute the F-factor for each detector in each of the fourteen RSB bands and at both HAM sides and in both high and low gains for dual gain bands. To give a few examples of the computed F-factors, in Fig. 4, we plot per orbit simple-averaged F-factors versus orbit number (each

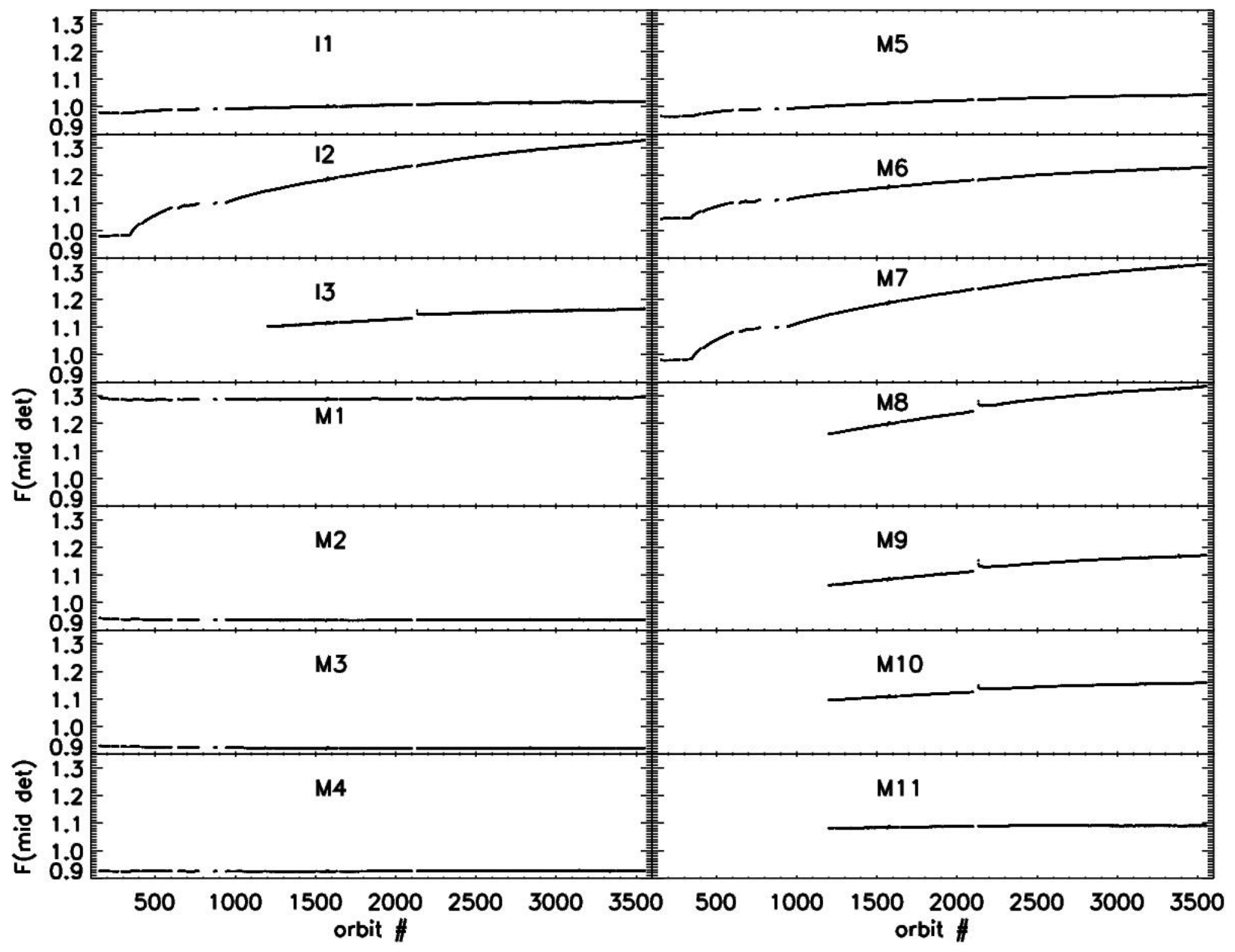

Fig. 4. F-factors versus orbit number for the mid-detectors of RSB bands at HAM side A and high gain for dual gain bands. 
orbit lasts about 101 minutes) for the mid-detector (detector 8 for the $\mathrm{M}$ bands and 16 for the I bands) of each of the RSB bands at HAM side A and high gain for dual gain bands. A major feature in Fig. 4 is that the F-factors for the M1 band are about 1.3, much larger than one. The reason is due to that in the prelaunch calibration to determine $\left(c_{0}, c_{1}, c_{2}\right)$, the RSR in the out-of-band region was not considered. The unusually large out-of-band RSR at wavelengths larger than that for the central peak for the M1 band, coupled with a radiance source redder than the Sun, leads to a computed spectral radiance smaller than what it should be. Consequently, $c_{1}$ for the M1 band is smaller than what it should be, yielding a large F-factor. The other major feature in Fig. 4 is that for bands I1, I2, M5, M6, and M7, the computed F-factors increase quickly with increasing orbit numbers after orbit 344 during which the NADIR door was opened, indicating degradation of optical components along the sensor's optical path. The F-factors for the SWIR bands also show such increase. Speculation on the cause of the degradation includes solar radiation damage. The radiation is mainly from the reflection of the solar radiation by the Earth through the EV port and in a small portion from the scattered solar radiation by the SD.

To help identify whether solar radiation is the root cause, the RTA was stowed for a few times, pointing away from the EV port. During the stows, no solar radiation entered the RTA. We plot in Fig. 5a, the VISNIR band middetector per orbit averaged F-factors normalized to the respective values at orbit 154 versus equivalent EV port solar radiation exposure time. In Fig. 5b, we plot the SWIR band mid-detector per orbit averaged F-factors normalized to the respective values at orbit 1201 versus the exposure time. The plots show smooth curves, revealing that solar radiation is very likely the cause of the degradation.

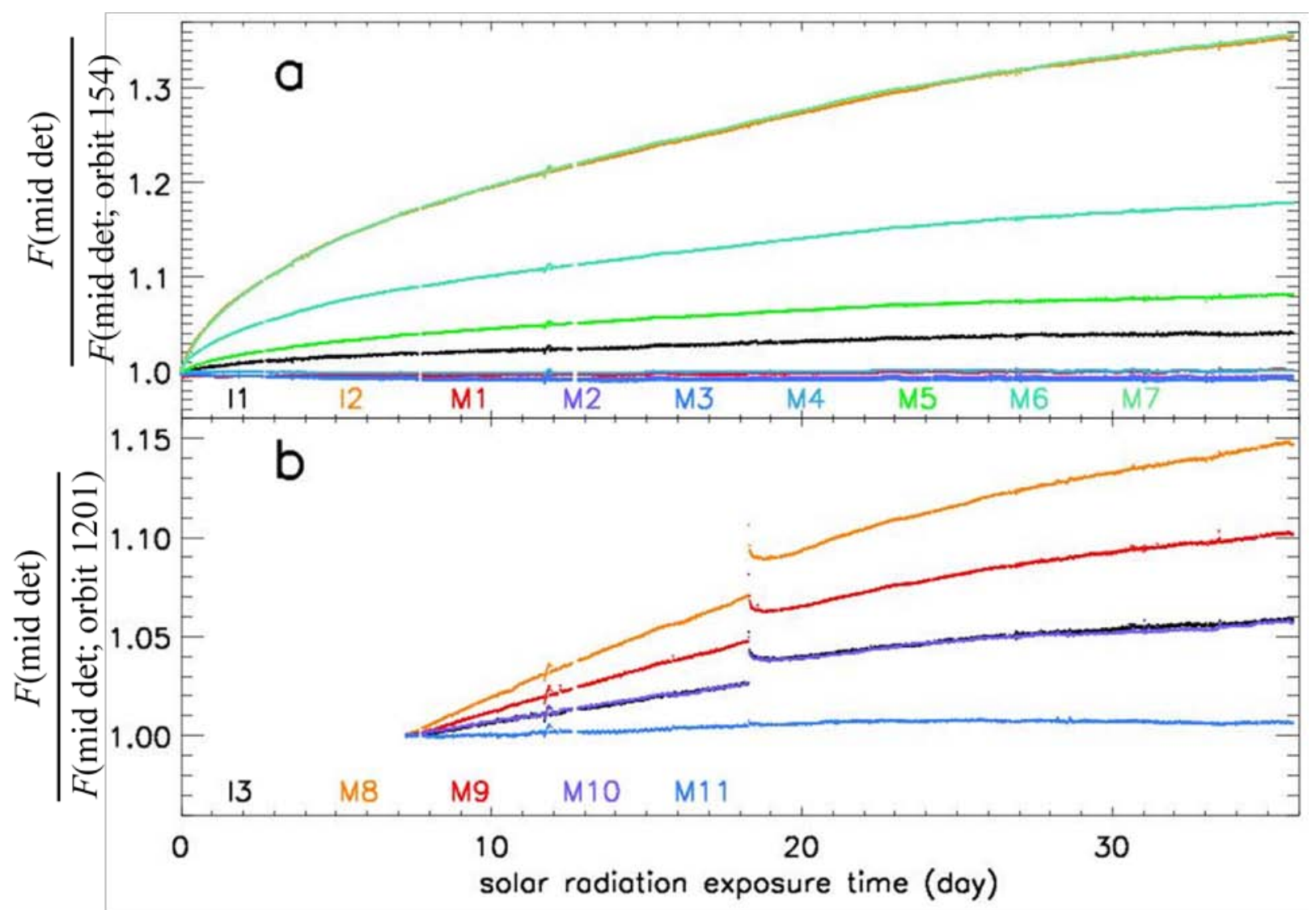

Fig. 5. Per orbit averaged normalized $F$ (mid det)/F(mid det; orbit 154) versus EV port solar radiation exposure time for the (a) VISNIR and (b) SWIR bands 
Reflectance and surface contamination measurements on the Telescope Witness Mirror (TWM) by the Aerospace Corp. indicate that the Rotating Telescope Assembly may be contaminated with tungsten oxides on its four reflective mirror surfaces. It is known that tungsten oxides, after exposure to UV radiation, become light absorbent over NIR and SWIR wavelengths ${ }^{5}$. Mathematical models to account for the observed F-factors, based on RTA mirror surface contamination, have been developed. The models and the applications of the models to address issues such as projected and smoothed F-factors, projected signal-to-noise ratio, and the impact of the RTA mirror darkening effect on the $R S R$ will be described elsewhere.

In addition to the unusually large F-factors for band M1 and the quick increase of the F-factors with increasing orbit numbers after orbit 344 for bands I1, I2, M5, M6, and M7, there are a few noticeable features for the F-factors. To help visualize the features, we plot in Figs. 6a-b, per orbit averaged normalized F-factors versus orbit number. First, the large deviations from the trend around orbits 1563 to 1577 are due to the yaw maneuvers of the spacecraft. In addition, for the SWIR bands, the discontinuities around orbits 2101 to 2133 and the unusually large F-factors right after orbit 2133 are due to the Spacecraft Control computer reset. The reason why the reset caused the behavior of the F-factors is under investigation. We also notice that many of the peaks and valleys in the F-factors are in sync across the bands. In particular, a hump is observed over orbits 1800 to 1900 for all bands, likely due to a solar storm.

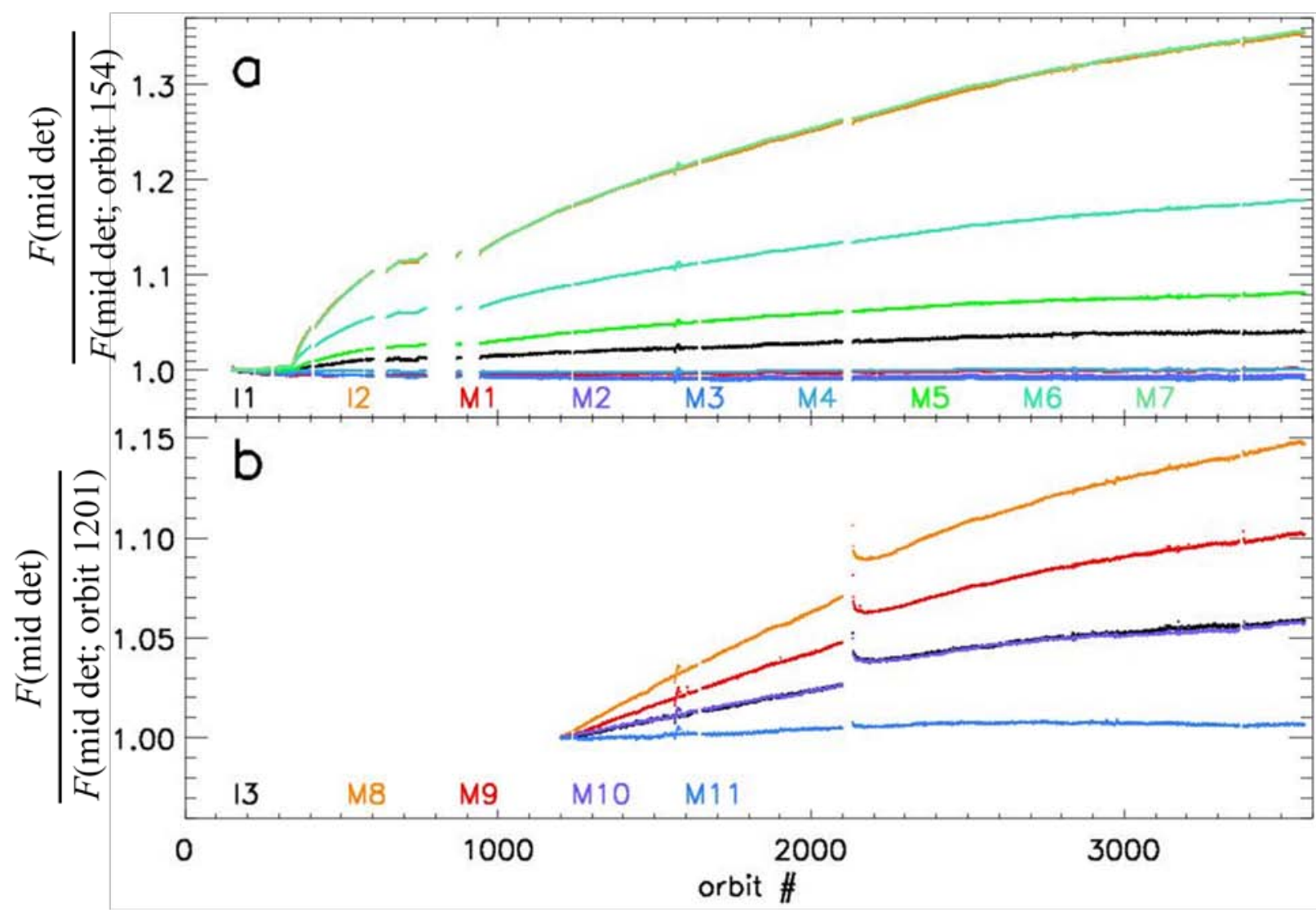

Fig. 6. Per orbit averaged normalized F(mid det)/F(mid det; orbit 154) versus orbit number for the (a) VISNIR and (b) SWIR bands

Finally, as revealed by Eqs. (2) and (6), there are many sources of error in the computed F-factor. For example, an error in $H\left(\lambda, t-t_{0}\right)$ would propagate into the computed F-factor. Quantitative descriptions of the uncertainty of the computed F-factor will be addressed elsewhere. 


\section{SUMMARY}

In this Proceeding, we described the methodology for the on-orbit radiometric response calibration through the SD for the Suomi NPP VIIRS RSB bands. We computed the F-factors for each detector in the RSB bands at both HAM sides and in both high and low gains for dual gain bands. The F-factors for the NIR and the SWIR bands increase quickly with time right after the NADIR door was opened in orbit 344, demonstrating unexpected degradation of some of the components along the sensor's optical path. Plots of the F-factors versus the EV port solar radiation exposure time yield smooth curves, indicating that the source of the degradation is likely the solar radiation, consistent with the contamination of tungsten oxides on the RTA reflective mirror surfaces. The computed F-factors were used to validate the results from the Aerospace Corp.

\section{ACKNOWLEDGEMENTS}

We'd like to thank Jason Cardema, Frank DeLuccia, and Kameron Rausch of Aerospace Corp. for fruitful discussions on issues such as the accuracy of the computed F-factors, clarifications of the Look-Up-Tables (which store prelaunch radiometric response coefficient values and other parameters) and of the definitions of the solar angle. We'd also like to thank Steve Mills and Lushalan Liao of Northrop Grumman for useful communications.

\section{REFERENCES}

[1] Moeller, C., McIntire, J., Schwarting, T., and Moyer, D., “VIIRS F1 "best” relative spectral response characterization by the Government Team”, Proc. SPIE Vol 8153, 81530K1-15 (2011).

[2] Xiong, X., Sun, J., Barnes, W., Salomonson, V., Esposito, J., Erives, H., and Guenther, B., "Multiyear On-Orbit Calibration and Performance of Terra MODIS Reflective Solar Bands”, IEEE Trans. Geosci. Remote Sens., Vol. 45(4), 879-889 (2007).

[3] Lei, N., Chiang, K., Oudrari, H., and Xiong, X., “A Maximum Likelihood approach to determine sensor radiometric response coefficients for NPP VIIRS reflective solar bands”, Proc. SPIE Vol 8153, 81530J1-11 (2011).

[4] Wu, A., McIntire, J., Xiong, X., De Luccia, F. J., Oudrari, H., Moyer, D., Xiong, S., and Pan, C., “Comparison of VIIRS Pre-Launch RVS Performance Using Results from Independent Studies”, Proc. SPIE Vol 8153, 81530L111 (2011).

[5] Bechinger, C., Herminghaus, S., Petersen, W., and Leiderer, P., "Dynamics of the photochromic effect of $\mathrm{WO}_{3}$ ”, Proc. SPIE Vol 2255, 467-472 (1994).

Ning.Lei@sigmaspace.com 